\title{
Terapia de laser de baja potencia en mucositis oral
}

\author{
Low-power laser therapy in oral mucositis \\ Florencio De La Torre ${ }^{1,2}$ b,c,d, Carlos Alfaro ${ }^{1, a}$
}

\section{RESUMEN}

La mucositis oral es una complicación frecuente del tratamiento antineoplásico, producto de efectos citotóxicos por la quimioterapia y radioterapia de cabeza y cuello,dependiendo de su severidad puede dificultar el habla, la deglución y predispone al paciente a deficiencias nutricionales, infecciones secundarias, sepsis grave, provocando alta morbilidad del paciente. La mayoría de investigadores concluyen que la terapia Laser de baja potencia es una alternativa de primera elección para la prevención y tratamiento de mucositis oral, comprobado en diversos estudios que estimula la producción de colágeno, elastina, proteoglicanos, la revascularización, entre otras propiedades que aceleran el proceso de cicatrización, además del efecto antiinflamatorio por inhibición de la COX2 así como no presentar efectos colaterales significativos, técnica atraumática y de costo bajo.

PALABRAS CLAVE: Mucositis oral, láser, quimioterapia, radioterapia.

\section{SUMMARY}

Oral mucositis is a common complication of cancer treatment, due to cytotoxic effects of chemotherapy and radiotherapy for head and neck, depending on its severity may hinder speech, swallowing and predisposes the patient to nutritional deficiencies, secondary infections, severe sepsis, causing high morbidity of the patient. Most researchers conclude that low-power laser therapy is an alternative of choice for the prevention and treatment of oral mucositis, proven in several studies that stimulates production of collagen, elastin, proteoglycans, revascularization, among other properties that accelerate the healing process, besides the anti-inflammatory effect by inhibiting COX-2 and not present significant side effects, atraumatic and inexpensive.

\section{KEY WORDS: Oral mucositis, laser,chemotherapy, radiotherapy}

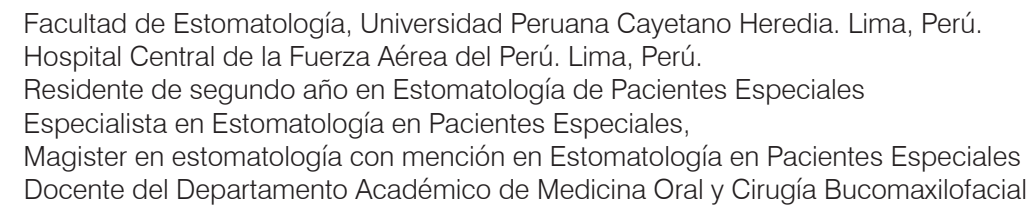




\section{INTRODUCCIÓN}

Los tratamientos antineoplásicos como quimioterapia (QT) y radioterapia ( RT) de cabeza y cuello así como en transplante de médula ósea (TMO) generan importantes complicaciones en la cavidad oral,la preservación del órgano bucal y mantención de las funciones como habla,deglución, son llevados en cuenta para la elección de la estrategia terapéutica a ser utilizada para combatir el cáncer (1).

Paraevitar o disminuir la resección quirúrgica se emplea la radioterapia preoperatoria combinada o no con la quimioterapia. La RT es ampliamente indicada en casos de neoplasias malignizadas de cavidad oral y puede ser terapia exclusiva para tumores de estadio inicial o puede ser una modalidad terapéutica en el post operatorio de vaciamiento de ganglios linfáticos cervicales, permitiendo evitar metástasis a través de la microcirculación regional comprometiendo la expectativa de cura y calidad de vida del paciente después del tratamiento (1-3).

Los cuidados básicos y precauciones deben ser tomados en cuenta antes, durante y después del tratamiento, entre estas complicaciones están la xerostomía, reacciones cutáneas,osteorradionecrosis y mucositis. La participación del cirujano dentista (CD)dentro del equipo multidisciplinario en el tratamiento de neoplasias malignas de la cavidad oral es fundamental asumiendo como responsabilidad realizar prevención, diagnóstico y tratamiento de estas alteraciones para evitar y/o disminuir riesgos de infección, acrecentar la condición de salud del paciente oncológico (3-5).

La mucositis oral (MO) constituye una de las complicaciones más comunes del tratamiento antineoplásico, es un cuadro inflamatorio de la mucosa oral producto de efectos citotóxicos por la QT y RT de cabeza y cuello, cuya evolución de las ulceraciones puede provocar alta morbilidad del paciente. El cuadro doloroso de las ulceraciones dificulta la deglución, habla, predispone al paciente a deficiencias nutricionales cuyo impacto puede ser alto durante el tratamiento oncológico $(5,6)$. Así mismo la pérdida de continuidad de la barrera de la mucosa oral predispone a infecciones secundarias que pueden provocar sepsis grave. Varias modalidades de tratamientos de la MO han sido propuestos pero todavía no hay un concenso en cuanto a la terapia más adecuada. La terapia con Laser de baja potencia(TLBP) ha destacado en los últimos años como una opción de prevención en el tratamiento de $\mathrm{MO}$, principalmente en función de sus propiedades como estímulo en la reparación de tejidos sin provocar efectos colaterales significati$\operatorname{vos}(7-9)$.

\section{Aspectos clínicos y factores de riesgo de mucositis oral}

El término "mucositis" respecto a su significado genérico es "inflamación de la mucosa" ha sido utilizado en la literatura científica para describir lesiones recurrentes por efectos citotóxicos provocados por la QT y RT de cabeza y cuello que se presentan en el tracto oral, esofágico, gastrointestinal $(10,11)$.

La incidencia de MO es mayor en pacientes en etapa pre transplante de medula ósea, en la terapia de infusión continua para cáncer de mama y colon y en la terapia para tumores de cabeza y cuello. Toda la mucosa oral puede ser afectada sin embargo los tejidos no queratinizados, como mucosa labial, mucosayugal, piso de boca, vientre de lengua y paladar blando son los más afectados (11).

\section{Aspectos clínicos de mucositis}

El aspecto clínico de la MO permite distinguir distintas fases de la lesión, que indica el grado de severidad.En las últimas cuatro décadas se han propuesto varios modelos evolucionados que documentan y cuantifican los cambios producidos en los tejidos epiteliales de la cavidad oral así como las alteraciones en la función, durante y después del tratamiento del cáncer (12).

Las escalas de valoración de la mucositis seclasifican en escalas "generales", compuestas por 4 o 5 variables y escalas con "múltiples variables"valoran variables diferentes, y su correspondencia con el estado de salud oral y la función; las puntuaciones obtenidas se suman y se obtiene una valoración global de la severidad de la mucositis (13). A esta clasificación debemos agregar las escalas específicas, en las que se considera el tipo de tratamiento que origina la mucositis.

Los sistemas de medición más empleados, son el sistema de la OMS y el del Instituto Nacional del Cáncer (NCI), estas escalas poseen simplicidad, lo que favo- 
rece su empleo, rápido y fácil (12).En 1979, la OMS definió el estado de las lesiones de la mucositis según la severidad de las mismas compuesta de 5 grados de normal a severo, estableciendo así grados del 0 a 4 (Tabla 1). En 1998 el NCI presentó una actualiza- ción de los CTC (Common Toxicity Criteria)(14), en la que se diferencia la mucositis debida a radiación, la mucositis debida a quimioterápicos, y la mucositis derivada del transplante de médula ósea, y que clasifica según la zona de aparición (Tabla 2).

Tabla 1. Escalas generales de valoración de la mucositis

\begin{tabular}{|c|c|c|c|c|c|}
\hline Escala & Grado 0 & Grado 1 & Grado 2 & Grado 3 & Grado 4 \\
\hline $\begin{array}{l}\text { FHCRC physician's } \\
\text { rating scale * }\end{array}$ & No mucositis & Mucositis Leve & $\begin{array}{l}\text { Mucositis mode- } \\
\text { rada }\end{array}$ & Mucositis severa & $\begin{array}{l}\text { Mucositis que } \\
\text { amenaza la } \\
\text { vida, mortal }\end{array}$ \\
\hline Lindquist/Hickey & No estomatitis & $\begin{array}{l}\text { Eritema } \pm \\
\text { ligero ardor }\end{array}$ & $\begin{array}{l}\text { Eritema y ulcera- } \\
\text { ción o } \\
\text { placas blancas; } \\
\text { dolor oral, } \\
\text { pero puede } \\
\text { comer. }\end{array}$ & $\begin{array}{l}\text { Eritema y ulce- } \\
\text { ración } \\
\text { o placas blancas; } \\
\text { dolor oral, } \\
\text { incapacidad para } \\
\text { comer. }\end{array}$ & \\
\hline $\begin{array}{l}\text { OMS** } \\
\text { WHO }\end{array}$ & $\begin{array}{l}\text { Normalidad } \\
\text { Normality }\end{array}$ & $\begin{array}{l}\text { Eritema } \\
\text { generalizado } \\
\text { Mucosa } \\
\text { enrojecida. } \\
\text { No dolor. } \\
\text { Voz normal }\end{array}$ & $\begin{array}{l}\text { Eritema. } \\
\text { Úlceras poco } \\
\text { extensas. } \\
\text { Se mantiene la } \\
\text { deglución de } \\
\text { sólidos. } \\
\text { Dolor ligero }\end{array}$ & $\begin{array}{l}\text { Úlceras extensas. } \\
\text { Encías edemato- } \\
\text { sas. } \\
\text { Saliva espesa. } \\
\text { Se mantiene la } \\
\text { capacidad de de- } \\
\text { glutir líquidos. } \\
\text { Dolor. } \\
\text { Dificultad para } \\
\text { hablar. }\end{array}$ & $\begin{array}{l}\text { Úlceras muy } \\
\text { extensas. } \\
\text { Encías sangrantes. } \\
\text { Infecciones. } \\
\text { No hay saliva. } \\
\text { Imposibilidad de } \\
\text { deglutir. } \\
\text { Soporte enteral o } \\
\text { parenteral. } \\
\text { Dolor muy ex- } \\
\text { tenso. }\end{array}$ \\
\hline
\end{tabular}

\section{Fisiopatología de la mucositis}

Hasta hace poco se describía la progresión de la MOen cuatro fases: fase inflamatoria y/o vascular, fase epitelial, fase ulcerativa y/o bacteriológica, fase curativa(15). Actualmente, la fisiopatologíade la enfermedad describe cinco fases, la primera fase de iniciación donde la QT y RTprovocan la ruptura de cintas de ADN y la producción de especies reactivas de oxigeno (ROS), a pesar que la mucosa parece absolutamente normal en esta fase su destrucción será provocada por una cascada de eventos que ocurren en la lámina propia.La fase de respuesta del daño prima- rio es la segunda fase de este proceso que consiste en la activación de factores de transcripción (NF-kB) y producción de citosinas pro inflamatorias(TNF- $\alpha$ y IL-1 $\beta$ ) por productos de la fase anterior. Lesión al tejido conjuntivo y al endotelio reducen la oxigenación epitelial, resultando en daño y muerte de células epiteliales. La fase siguiente de amplificación de señales promueve una realimentacion o feedback positivo de citosinas proinflamatorias y amplifican el daño iniciado por la radiación o agente quimioterápico (6). Sigue la fase ulcerativa cuando hay pérdida de la integridad de la mucosa,estas lesiones extrermadamente dolorosas son de gran importancia, pues funcionan como 
Tabla 2. Escalas específicas de tratamiento

\begin{tabular}{|c|c|c|c|c|c|c|}
\hline Escala & $\begin{array}{c}\text { Terapia } \\
\text { antineoplá- } \\
\text { sica }\end{array}$ & Grado 0 & Grado 1 & Grado 2 & Grado 3 & Grado 4 \\
\hline NCI-CTC & QT & Ninguno & $\begin{array}{l}\text { No dolor, úlceras,eritema, } \\
\text { o dolor leve en ausencia } \\
\text { de lesiones }\end{array}$ & $\begin{array}{l}\text { Dolor, eritema, edema } \\
\text { o úlceras, pero puede } \\
\text { comer o tragar. }\end{array}$ & $\begin{array}{l}\text { Dolor, eritema, } \\
\text { edema o úlceras. } \\
\text { Requiere hidratación } \\
\text { intravenosa. }\end{array}$ & $\begin{array}{l}\text { Ulceración } \\
\text { severa o requiere } \\
\text { nutrición enteral } \\
\text { o parenteral } \\
\text { o intubación } \\
\text { profiláctica. }\end{array}$ \\
\hline NCI-CTC & RT & Ninguno & Eritema mucoso & $\begin{array}{l}\text { Lesiones } \\
\text { Pseudo membranosa } \\
\text { irregulares, } \\
\text { generalmente de } 1,5 \\
\mathrm{~cm} \text {. de diámetro y no } \\
\text { contiguas. }\end{array}$ & $\begin{array}{l}\text { Lesiones } \\
\text { Pseudo membranosas- } \\
\text { confluyentes, y gene- } \\
\text { ralmente mayores de } \\
1,5 \mathrm{~cm} \text {. de diámetro. }\end{array}$ & $\begin{array}{l}\text { Necrosis o } \\
\text { ulceraciones } \\
\text { profundas, puede } \\
\text { incluir sangrado } \\
\text { no inducido por } \\
\text { traumas menores } \\
\text { o abrasiones. }\end{array}$ \\
\hline NCI-CTC & TMO & Ninguno & $\begin{array}{l}\text { No dolor, úlceras, } \\
\text { eritema, o dolor leve en } \\
\text { ausencia de lesiones }\end{array}$ & $\begin{array}{l}\text { Dolor, eritema, edema } \\
\text { o úlceras, pero puede } \\
\text { tragar. }\end{array}$ & $\begin{array}{l}\text { Dolor, eritema, ede- } \\
\text { ma o úlceras, que } \\
\text { dificultan tragar o que } \\
\text { requieren hidratación } \\
\text { enteral o parenteral, o } \\
\text { soporte nutricional. }\end{array}$ & $\begin{array}{l}\text { Ulceraciones } \\
\text { severas que pre- } \\
\text { cisan intubación } \\
\text { preventiva o que } \\
\text { resultan en una } \\
\text { pulmonía por } \\
\text { aspiración. }\end{array}$ \\
\hline WCCNR & QT & $\begin{array}{l}\text { Ninguna } \\
\text { lesiónColor } \\
\text { Rosa }>50 \% \\
\text { Sangrado: } \\
\text { Ninguno }\end{array}$ & $\begin{array}{l}\text { Lesiones: } 1-4 \\
\text { Color : Ligeramente } \\
\text { Rojo }>50 \%\end{array}$ & $\begin{array}{l}\text { Lesiones: }>4 \\
\text { Color moderadamente } \\
\text { Rojo }>50 \% \\
\text { Sangrado con la comi- } \\
\text { da o la higiene }\end{array}$ & $\begin{array}{l}\text { Lesiones: Concluyen- } \\
\text { tes en un } 50 \% \text { o } \\
\text { más de la superficie } \\
\text { mucosa } \\
\text { Color muy Rojo > } \\
50 \% \text {. } \\
\text { Sangrado espontáneo, } \\
\text { sangre fresca, o apari- } \\
\text { ción de sangre seca en } \\
\text { la almohada }\end{array}$ & \\
\hline $\begin{array}{l}\text { RTOG/ } \\
\text { EORTC }\end{array}$ & RT & Sin cambios & $\begin{array}{l}\text { Eritema } \\
\text { Dolor leve que no } \\
\text { requiere analgesia. }\end{array}$ & $\begin{array}{l}\text { Mucositis parcheada. } \\
\text { Sangrado inflamatorio. } \\
\text { Dolor moderado que } \\
\text { requiere analgesia. }\end{array}$ & $\begin{array}{l}\text { Mucositis } \\
\text { confluyente. Dolor } \\
\text { severo }\end{array}$ & $\begin{array}{l}\text { Ulceración. } \\
\text { Hemorragia. } \\
\text { Necrosis. }\end{array}$ \\
\hline $\begin{array}{l}\text { Escala de } \\
\text { Spijkervet. }\end{array}$ & TMO & No mucositis & $\begin{array}{l}\text { Decoloración blanquecina } \\
\text { Superficie: }<1 \mathrm{~cm} \text {. }\end{array}$ & $\begin{array}{l}\text { Eritema } \\
\text { Superficie: } 1-2 \mathrm{~cm} .\end{array}$ & $\begin{array}{l}\text { Pseudomembranas } \\
\text { Superficie: } 2-4 \mathrm{~cm} .\end{array}$ & $\begin{array}{l}\text { Ulceración } \\
\text { Superficie: > } \\
4 \mathrm{~cm} \text {. }\end{array}$ \\
\hline
\end{tabular}

*Áreas evaluadas: mucosa yugal derecha e izquierda, paladar duro y blando, dorso lingual, lateral de la lengua (derecho e izquierdo), piso de la boca. QT: Quimioterapia

RT: Radioterapia

TMO: Transplante de médula ósea. 
puerta de entrada para bacterias principalmente en pacientes con neutropenia, finalmente la fase de curación corresponde a la migración, proliferación y diferenciación de células epiteliales, caracterizado por la resolución de la lesiones de la mucosa oral $(16,17)$. Clínicamente la MO inicia con manifestación de eritema, seguida de descamación de epitelio y ulceración del mismo. Eventualmente con presencia de exudado fibrinoso, formación de una pseudo membrana sobre la úlcera, pérdida de epitelio que expone el tejido conjuntivo subyacente, causando dolor y disconfort durante el tratamiento antineoplásico(17) (Figuras1, 2 y 3).

La sintomatología recurrente dolorosa de la mucositis ulcerativa interfiere en la calidad de vida y nutrición del individuo afectado, así como pérdida significativa de peso, a veces es necesario instalar sonda nasogástrica para alimentación enteral. La disrupción de labarrera dela mucosa aumenta el riesgo de infecciones y limita la dosis de laterapia la cual puedeser alterada en su intensidad o interrumpida en casos severos de mucositis, perjudicando el control de la neoplasia maligna o el pronóstico del paciente. El aumento de la morbilidad y el costo operacional del tratamientorequieren una terapia efectiva en el control de la MO (17).

\section{Terapias empleadas en el tratamiento de la mu- cositis}

Aún no hay consenso en la comunidad científica deuna terapia efectiva aprobada para la $\mathrm{MO}$, sin embargo se pretende la reducción de síntomas como alivio de dolor, utilizando analgésicos narcóticos.Las terapias farmacológicas utilizadas actualmente para $\mathrm{MO}$ incluyen citoprotectores, factores de crecimiento celular, vitamina E, suplementos metabólicos, antibióticos, antimicrobianos, agentes lubrificantes,antiinflamatorios y terapia génica (18).

Amifostina(Ethyol) (18) es un citoprotector directo y se muestra eficiente para reducir la mucosistis inducida por quimio o radioterapia. Su desventaja es la administración endovenosa, posee fuertes efectos colaterales como nauseas, vómitos, hipotensión para los cuales debe ser administrada una droga antiemética concomitante $(19,20)$.

Palifermina (Kepivance) es un factor de crecimiento de queratinocitos (KGF) responsable de estimular proliferación, migración y diferenciación de epitelio. El KGF endógeno normalmente es producido por fibroblastosy expresada exclusivamente en células epiteliales de una variedad de tejidos, por lo tanto sigue siendo cuestionable la acción de este fármaco en células neoplásicas malignas a pesar de que los estudios sean favorables (21).

El uso de colutorios con clorhexidina, antibiótico de amplio espectro aún no ha sido totalmente elucidado en MO inducida por RT. Otras soluciones empleadas en los cuidados de higiene oral en pacientes con alto riesgo de infecciones a pesar de promover mayor confort del paciente son paliativos por que no poseen efecto significante en mucositis (22).

Benzidamina es un antiinflamatorio no esteroideoindicado para atenuación de una serie de condiciones inflamatorias, incluyendo MO inducido por radiación antineoplásica o QT. Estudios en vivo constataron la reducción de niveles plasmáticos de TFN- $\infty$ e IL- $1 \beta$ por la acción de la benzidamina estas citosinas son
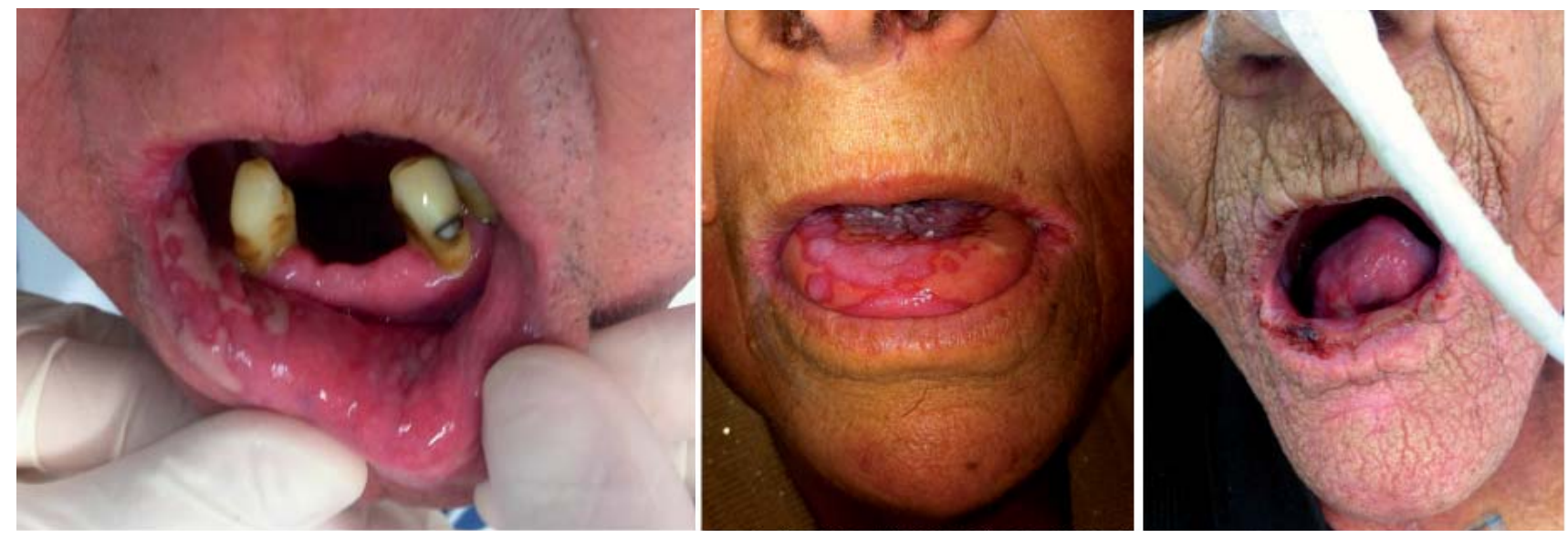

Figura 1, 2 , 3. Mucositis Oral grado 3 según la OMS (Cortesía del Dr. Florencio De La Torre) 
encontradas en exceso en la mucosa afectada por mucositis y en infecciones por Cándida albicans, principal microorganismo agravante de mucositis oral. Por ser considerado un eficiente analgésico y antiinflamatorio supresor de citosinas, puede ser usado para atenuar el dolor y severidad de MO (23).

\section{Terapia laser de baja potencia}

Láser es un acrónimo en Inglés, (Light Amplification by Stimulated Emission of Radiation) significa amplificación de luz por emisión estimulada de radiación (24). Se trata de una radiación electromagnética con características propias que difieren de una luz común porque tiene una sola longitud de onda que se propaga coherentemente en el espacio y tiempo, concentraciones colimadas y niveles altos de energía (25).

Existen diversos tipos de láser que son empleados en estomatología y estos se clasifican en Láser de alta potencia o quirúrgico (HILT) tienen capacidad de corte, coagulación, cauterización, ablación de efectos en preparaciones de cavidades por caries dental y el Láser de baja potencia o laser terapéuticos (LILT) $(25,26)$.

La TLBP es una alternativa efectiva para prevenir y tratarla MO inducida por RT, QT y TMO. Esta terapéutica consiste en la interacción de la luz de baja energía a pocos Jouls por centímetro cuadrado, con las células y tejidos, provocando efectos fotoquímicos, fotofísicos y fotobiológicos, sin causar aumento de la temperatura por encima de $98^{\circ} \mathrm{F}$, tiene efecto biomodulador sobre las células y los tejidos debido a la absorción de la energía de la luz por los fotorreceptores endógenos (Figuras 4 y 5 ).

En 1989, la revisión de estudios in vitro, revelaron que los receptores fotoactivados son componentes principales de la cadena respiratoria celular, la activación de estos receptores podrían conducir a la estimulación o inhibición del metabolismo celular dependiendo de la dosis de energía empleada considerando que dosis bajas de energía podrían regular la producción de especies oxígeno reactivo. La luz visible puede llevar a cambios fotoquímicos en los fotorreceptores de la mitocondria estimulando a su actividad, alterando el metabolismo celular y produciendo el efecto de transducción en otros componentes de la

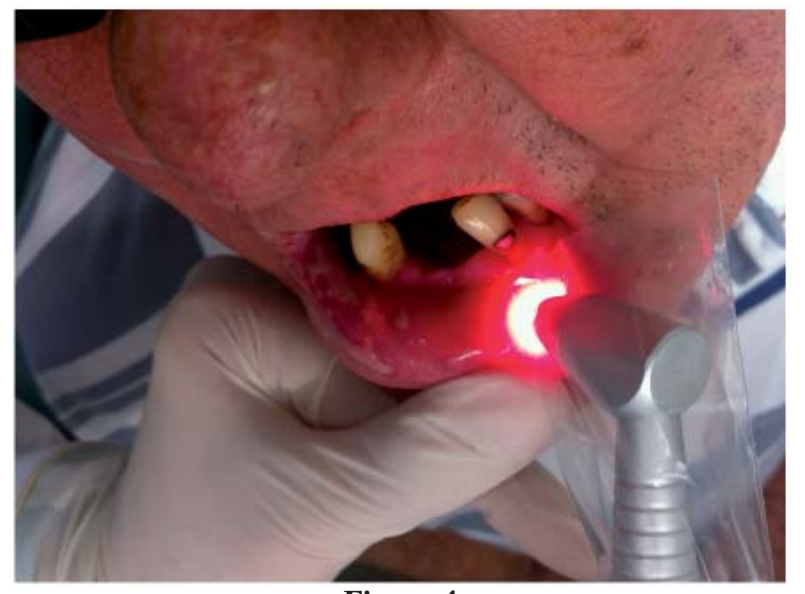

Figura 4

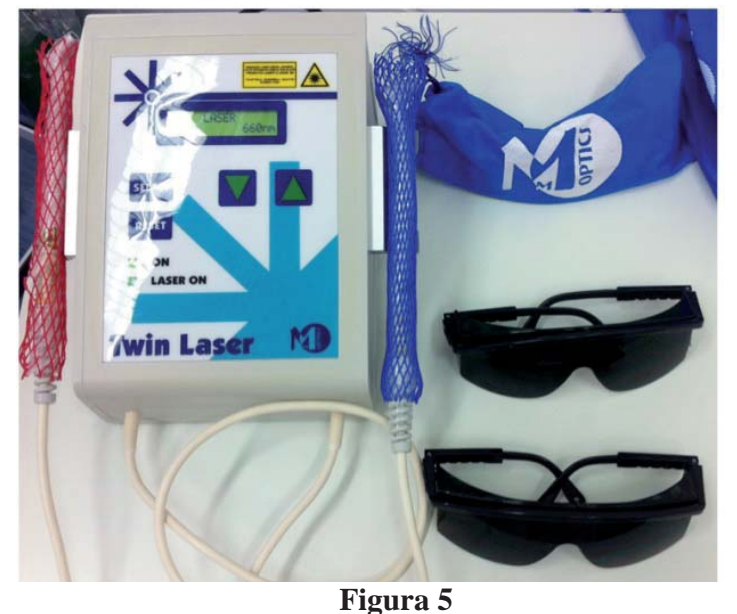

célula (efecto biomodulador), otros sugieren que este efecto es debido a los cambios en la fotofísica de los canales de $\mathrm{Ca}++$ en la membrana celular $(26,27)$.

Los estudios en modelos animales de la MO han comprobado que TLBP promueve la cicatrización de heridas, así como un efecto anti-inflamatorio a través de la inhibición de la COX-2, evidenciado por la reducción de infiltrado de neutrófilos y la expresión de la ciclooxigenasa-2 $(26,27)$. Otros eventos biológicos producidos por la TLBP son la proliferación epitelial y estimulación de fibroblastos induciendo su maduración, locomoción y transformación en miofibroblastos. Estimula la producción de colágeno, elastina y proteoglicanos, revascularización, contracción de la herida, el aumento de la fagocitos macrófagos, aumento de la proliferación y activación de los linfocitos y resistencia a la tracción, lo que acelera el proceso de curación (27). 
Los estudios de laboratorio indican que los mecanismos de la TLBP en tejidos, tienen un mejor efecto cuando son emitidos desde la región del rojo visible a infrarrojo cercano (27). También recomiendan emplear láser con poder de penetración baja, longitudes de onda entre 640-940 nm, y que la aplicación se lleve a cabo de manera oportuna sobre la lesión, la energía será absorbida solamente por el tejido adyacente al punto de aplicación, y una fina capa más allá del punto alcanzado por la radiación. El diodo que emite luz roja visible tiene menos poder penetración, siendo más adecuada para la reparación de tejidos, mientras que el diodo con longitud de onda más larga, y que por lo tanto, emite láser infrarrojo, tiene mayor capacidad para penetración, siendo su indicación para analgesia (28).El láser de He-Ne (helio-neón) y GaAlAs (aluminio-galio Arsénico) son también los más indicados por los buenos resultados en MO $(27,28)$.

Figueiredo ALP. y cols. demostraron que la TLBP en pacientes sometidos a oncoterapia es aproximadamente nueve veces más eficaz en la prevención de MO grado $>3$ que en los pacientes sin tratamiento con láser. Comprobaron el efecto profiláctico significativo de $\mathrm{MO}$ de grado $>3$, y recomiendan realizar estudios con muestras de mayor tamaño para una mejor evaluación del efecto profiláctico. Rampini y col., proponen a la TLBP como alternativa eficaz para prevenir y tratar la mucositis oral, debido a ser no traumático, bajo costo y buenos resultados. Estudios aleatorizados con pacientes sometidos a trasplante de médula ósea y radioterapia en la cabeza y cuello confirmaron la disminución de la incidencia de mucositis oral y dolor durante período de tratamiento $(27,28)$.

Pinto comparó dos protocolos diferentes: terapéuticos (grupo 1) y profilácticos-terapéuticos (grupo 2) en 14 pacientes utilizando láser de diodo (medio activo: InGaAIP) en quienes evaluó el cambio del antes y después de la TLBP en diferentes grados de MO. El grupo 2 mostró un menor grado de mucositis y redujo la remisión así como la intensidad del dolor comparado con el grupo 1(24).

Cavalcanti y col., en su revisión de la literatura afirman que la TLBP es una opción de tratamiento excelente, ya que tiene efectos beneficiosos para tejidos irradiado (25).

La Multinacional Asociación de Tratamiento de So- porte en Cáncer / Sociedad Internacional de Oncología Oral (MASCC / ISOO) realizó una revisión sistemática y recomienda usar TLBP como prevención de $\mathrm{MO}$ en pacientes adultos que hayan recibido TMO con altas dosis de quimioterapia con o sin radiación total del cuerpo, empleando $650 \mathrm{~nm}$ de longitud de onda, $40 \mathrm{~mW}$ de potencia por cada centímetro cuadrado y la dosis de energía de $2 \mathrm{~J} / \mathrm{cm} 2(2 \mathrm{~s} /$ punto) y en los casos de pacientes sometidos a radioterapia sin quimioterapia concomitante emplear una longitud de onda alrededor de $632,8 \mathrm{~nm}$ (24).

Kelner y Freire Lisboa de Castro informan la eficiencia de láser de baja potencia para el tratamiento de MO inducida por la radiación en dos casos clínicos del Hospital de Cáncer de Pernambuco (29).

\section{DISCUSIÓN}

No hay un consenso propuesto para el tratamiento de $\mathrm{MO}$, los tratamientos más usados son paliativos y ninguno tiene efectividad terapéutica comprobada, se emplean analgésicos narcóticos, citoprotectores, factores de crecimiento celular, vitamina E, suplementos metabólicos, antibióticos, agentes lubricantes, antiinflamatorios y terapia génica. Contrariamente la TLBP es eficaz en el tratamiento y prevención de $\mathrm{MO}$, estos efectos ampliamente descritos en la literatura así como en los diversos artículos analizados en esta publicación son confirmados por Migloriati en su revisión sistemática, en que la luz visible puede llevar a cambios fotoquímicos en los receptores de la mitocondria estimulando su actividad, comprobado en estudios en modelos animales que la TLBP promueve la cicatrización de las heridas así como su efecto antiinflamatorio entre otros efectos biológicos que reporta Rampini y col. en utilización de LBPT para prevención de $\mathrm{MO}$ como proliferación epitelial ,estimulación de fibroblastos induciendo su maduración, locomoción y transformación en miofibroblastos estimula la producción de colágeno y elastina ,revascularización contracción de la herida aumento de fagocitosis de la proliferación y activación de los linfocitos lo que acelera el proceso de curación.

Para Figueiredo y col., en su revisión sistemática de láser terapia en control de MO; afirman que, LTBP es aproximadamente nueve veces más eficaz que en los pacientes sin tratamiento, comprobando el efecto 
profiláctico significativo de Mo grado 3 , sin embargo recomiendan realizar estudios con muestras de mayor tamaño para una mejor evaluación del efecto profiláctico.coincidiendo con los resultados del trabajo de Pinto T. que comparó dos protocolos diferentes en 14 pacientes utilizando láser de diodo y evaluaron el cambio del antes y después de la TLBP encontrando un menor grado de mucositis y reducción de la remisión así como, la intensidad del dolor.

\section{CONCLUSIONES}

La MO constituye una de las complicaciones más comunes del tratamiento antineoplásico, el dolor de las úlceras dificulta la deglución, habla y predispone al paciente a deficiencias nutricionales, infecciones secundarias generando sepsis grave.

La MO aumenta el riesgo de infecciones y limita la dosis de la QT - RT alterando su intensidad o interrumpiéndola en casos severos, perjudicando el control de la neoplasia maligna o el pronóstico del paciente aumentando la morbilidad y el costo del tratamiento.

La TLBP tiene efectos beneficiosos sobre los tejidos como activación de microcirculación, producción de capilares, antiinflamatoria y analgésico, estimular el crecimiento celular acelerando el proceso de cicatrización sin provocar efectos colaterales significativos.

La TLBP es una alternativa de primera elección siendo un método muy eficaz para prevenir y tratar los efectos secundarios e indeseables de la RT, QT como $\mathrm{MO}$, debe tener una longitud de onda entre $640 \mathrm{~nm}$ a $940 \mathrm{~nm}$, ser de penetración baja y usar un diodo de Luz roja visible que tiene la propiedad de reparar tejidos.

La participación del cirujano dentista dentro del equipo multidisciplinario en el tratamiento de neoplasias malignas es fundamental asumiendo como responsabilidad realizar prevención, diagnóstico y tratamiento de estas alteraciones para evitar y/o disminuir la probabilidad de infección yacrecentar la condición de salud del paciente oncológico.

\section{Correspondencia:}

Florecio de la Torre Moreno

Correo electrónico: florencio.de.la.torre@upch.pe

\section{REFERENCIAS BIBIOGRÁFICAS}

1. Cernea CR, Hojaij FC. Factores prognosticos. En: Cáncer de Boca: Aspectos Básicos e terapéuticos. 1ra Ed. Brasil: Elsevier; 2000. p.101-106.

2. Silva A, Simoes M, Nuñez SC. Laser de baixa Potencia principios básicos e aplicacoes clínicas naodontología. 1ra Ed. Río de Janeiro: Elsevier; 2012. p.105-116.

3. de Paula Eduardo C. Fundamentos de Odontologia - Lasers em Odontologia. 3ra ed. Río de Janeiro: Guanabara Koogan; 2010.p. 62-67.

4. Migliorati C, Massumoto C, Eduardo FP, et al. Low-energy Therapy in oral mucositis. J Oral Laser Applications. 2001;1(2):97-101.

5. Nes AG, Posso MB. Patients with moderate chemotherapy-induced mucositis: pain Therapy using low intensity lasers. Int Nurs Rev. 2005;52(1):68-72.

6. Filicko J, Lazarus HM, Flomemberg N. Mucosa injury in patients urdergoing hematopietic progenitor cell transplantation: new approaches to prophylaxis and treatment. Bone Marrow Transplantation. 2003;31:1-10.

7. Simoes A, Eduardo FP, Luiz AC, et al. Laser phototherapy as topical prophylaxis again head and neck cancer radiotherapy-induced oral mucositis; comparison between low and righ/low power laser. Lasers Surg Med. 2009;27(2);371374.

8. Jaguar GC, Prado JD, Nishimoto IN, et al. Lowenergy laser therapy for prevention of oral mucositis in hematopoietic stem cell transplantation. Oral diseases. 2007; 13(6): 538543.

9. Wong, SF, Wilder-Smith P. Pilot study of laser effects on oral mucositis in patients receiving chemotherapy. The Cancer Journal. 2002; 8(3): 247-254.

10. Peterson DE, Keefe DM, Hutchins RD, Schubert MM. Alimentary tract mucositis in cancer patients: impact of terminology and assessment on research and clinical practice. Supportive care in cancer. 2006; 14(6): 499-504.

11. Raber-Durlacher JE, Elad S, Barasch A. Oral mucositis. Oral oncology. 2010; 46(6): 452-456.

12. López-Castaño F, Oñate-Sánchez RE, RoldánChicano R., Cabrerizo-Merino MC. Measurement of secondary mucositis to oncohematologic 
treatment by means of different scale. Review. Medicina oral, patologia oral y cirugiabucal. 2004; 10(5): 412-421.

13. Schubert MM. Measurement of oral tissue damage and mucositis pain. Current and emerging issues in cancer pain: research and practice. 1993; $247-$ 265.

14. National Cancer Institute. Cancer Therapy Evaluation Program Common Toxicity Criteria; Version 4.0. Bethesda: National Cancer Institute; 1999

15. Sonis ST. Mucositis as a biological process: a new hypothesis for the development of chemotherapyinduced stomatotoxicity. Oral oncology.1998; 34(1): 39-43.

16. Sonis ST. The pathobiology of mucositis. Nature Reviews Cancer. 2004; 4(4): 277-284.

17. Sonis ST, Peterson RL, Edwards LJ, et al. Defining mechanisms of action of interleukin-11 on the progression of radiation-induced oral mucositis in hamsters. Oral Oncology. 2000; 36(4): 373-381.

18. Dörr W, Spekl K, Farrell CL. The effect of keratinocyte growth factor on healing of manifest radiation ulcers in mouse tongue epithelium. Cell proliferation. 2002; 35(S1): 86-92.

19. Ning S, Shui C, Khan WB, Benson W, Lacey DL, Knox SJ. Effects of keratinocyte growth factor on the proliferation and radiation survival of human squamous cell carcinoma cell lines in vitro and in vivo. International Journal of Radiation Oncology Biology Physics. 1998; 40(1): 177-187.

20. Wadleigh RG, Redman RS, Graham ML, Krasnow $\mathrm{SH}$, Anderson A, Cohen MH. Vitamin $\mathrm{E}$ in the treatment of chemotherapy-induced mucositis. The American journal of medicine.1992; 92(5): 481-484.

21. Jebb SA, Osborne RJ, Maughan TS, et al. 5-fluorouracil and folinic acid-induced mucositis: no effect of oral glutamine supplementation. British journal of cancer. 1994; 70(4): 732.

22. Symonds RP, McIlroy P, Khorrami J, et al. The reduction of radiation mucositis by selective decontamination antibiotic pastilles: a placebocontrolled double-blind trial. British journal of cancer. 1996; 74(2): 312.

23. Foote RL, Loprinzi CL, Frank AR, et al. Randomized trial of a chlorhexidine mouthwash for alleviation of radiation-induced mucositis. Journal of Clinical Oncology. 1994; 12(12): 26302633.

24. Pinto TAS. Efeito do laser de baixa intensidade na prevenção e tratamento da mucosite bucal. Rio grande: Pontifícia universidade católica do rio grande do sul; 2010.

25. Cavalcanti, TM, Almeida-Barros RQD, Catão
MHCD, Feitosa APA, Lins RDAU. Conhecimento das propriedades físicas e da interação do laser com os tecidos biológicos naodontologia; Knowledge of the physical properties and interaction of laser with biological tissue in dentistry. An bras Dermatol. 2011; 86(5): 955960.

26. Migliorati C, Hewson I, Lalla RV, et al. Systematic review of laser and other light therapy for the management of oral mucositis in cancer patients. Support Care Cancer. 2013;21(1): 333-341.

27. Rampini MP, Ferreira EMS, Ferreira CG, Antunes HS. Utilização da terapia com laser de baixapotência para prevenção de mucosite oral: revisão de literatura. Rev Bras Cancerol. 2009; 55(1): 59-68.

28. Figueiredo ALP, Lins L, Cattony AC, Falcão AFP. Laser therapy in oral mucositis control: a meta-analysis. Revista da Associação Médica Brasileira. 2013; 59(5): 467-474.

29. Kelner N, Freire Lisboa de Castro JO. Utilização do laser de baixapotência e do gluconato de clorexidinanaprevenção da mucosite oral em pacientes submetidos a radioterapia e/ou quimioterapia no Hospital do Câncer de Pernambuco. Dissertacao. Pernambuco, Recife: Universidade Federal de Pernambuco; 2006.

Recibido : 05/12/2015

Aceptado: 09/02/2016 